\title{
Mesenchymal stem cells confer resistance to doxorubicin-induced cardiac senescence by inhibiting microRNA-34a
}

\author{
WENZHENG XIA $^{1}$ and MENG HOU ${ }^{2}$ \\ Departments of ${ }^{1}$ Neurosurgery and ${ }^{2}$ Radiation Oncology, First Affiliated Hospital, \\ Wenzhou Medical University, Wenzhou, Zhejiang 325000, P.R. China
}

Received March 9, 2017; Accepted November 10, 2017

DOI: $10.3892 / \mathrm{ol} .2018 .8438$

\begin{abstract}
Doxorubicin (DOXO) is a chemotherapeutic agent widely used in the treatment of various types of cancer. However, cardiotoxicity is a major side effect of DOXO therapy due to the ability of this compound to induce cardiac cellular senescence. It is well known that microRNA (miR)-34a serves a role in cardiac dysfunction and ageing, and that it is involved in several cellular processes associated with DOXO-induced cardiotoxicity. Furthermore, mesenchymal stem cell (MSC)-based therapies have been reported to modulate cellular senescence. In the present study, the Transwell system was used to co-culture H9c2 cells and MSCs, and cell proliferation and viability were assessed. The expression of senescence-related genes, p53 and p16, and telomere length were analyzed using reverse transcription-quantitative polymerase chain reaction (PCR), and the protein expression levels of situin 1 (SIRT1) were detected by western blotting. Additionally, telomerase activity of H9c2 was examined using the Telo TAGGG Telomerase PCR ELISA PLUS kit. The present study revealed that, in the presence of DOXO, $\mathrm{H} 9 \mathrm{c} 2$ cells were in senescence, as characterized by a low proliferation rate, poor viability and a marked increase in the expression of p53 and p16. By contrast, when co-cultured with MSCs in the presence of DOXO, the proliferation and viability of $\mathrm{H} 9 \mathrm{c} 2$ cells increased. Additionally, the expression of p53 and p16 decreased, and increased length of telomere and telomerase activity was also observed. Additionally, the mechanism underlying the anti-senescence function of MSCs was revealed to involve the miR-34a-SIRT1 axis, confirmed by overexpressing miR-34a using a miR-34a mimic or silencing SIRT1 using small interfering RNA, which abolished the anti-senescence effect of MSCs on DOXO-treated $\mathrm{H} 9 \mathrm{c} 2$ cells. Taken together, the results of the present study
\end{abstract}

Correspondence to: Dr Meng Hou, Department of Radiation Oncology, First Affiliated Hospital, Wenzhou Medical University, 2 Fuxue Lane, Wenzhou, Zhejiang 325000, P.R. China

E-mail: 244517813@qq.com

Key words: doxorubicin, cardiac senescence, mesenchymal stem cells, microRNA-34a, sirtuin 1 suggest that MSCs may rejuvenate $\mathrm{H} 9 \mathrm{c} 2$ cells from a state of DOXO-induced senescence by increasing SIRT1 expression, there by inhibiting miR-34a. Therefore, treatment with MSCs may have important therapeutic implications in the restoration of cardiotoxicity in patients with cancer undergoing treatment with DOXO.

\section{Introduction}

Despite its adverse side effects, chemotherapy has made an essential contribution to cancer treatment in recent decades (1,2). Anthracyclines, a class of chemotherapeutic drug that have been widely used to treat several types of cancer, are among the leading causes of cardiotoxicity in cancer survivors (3). In particular, the anthracycline, doxorubicin (DOXO), demonstrates great therapeutic potential in a range of types of cancer. However, cardiotoxicity is a major limiting factor of DOXO therapy, with a spectrum of short- and long-term cardiotoxic effects induced by this compound, ranging from cardiomyocyte senescence and subclinical ventricular dysfunction to severe cardiomyopathy and heart failure that may result in cardiac transplantation or mortality $(4,5)$. Therefore, there is an urgent requirement to identify an efficient way to ameliorate DOXO-induced toxicity in order to prevent future cardiac complications.

Cell-based therapies have huge potential in the treatment of cardiovascular diseases, particularly bone marrow derived-mesenchymal stem cells (BM-MSCs), due to their regenerative properties and known bio-safety (6). In a rat model of DOXO-induced dilated cardiomyopathy, intravenous administration of BM-MSCs not only improved cardiac contractility, but also reduced myocardium fibrosis and left ventricular diameter (7). Additionally, it has been reported that the local administration of BM-MSCs after 2 weeks of DOXO treatment generated a marked improvement in left ventricular ejection fraction (8). Therefore, MSCs possess numerous characteristics that make them a promising tool for preventive or regenerative myocardium treatment, in an effort to prevent DOXO-induced cardiomyopathy. However, the mechanisms through which MSCs reduce DOXO-induced cardiomyocyte damage remain unclear.

MicroRNAs (miRs) are small noncoding RNA molecules that are critical for a large array of cellular processes, including survival and growth in both pathological and non-pathological 
conditions $(9,10)$. Among them, the miR-34 family is associated with the cardiovascular system. Indeed, miR-34 family members (miR-34a, -34b and -34c) are upregulated in the heart in response to stress, including myocardial infarction (11), and they regulate cardiac ageing and function (12). In particular, miR-34a modulates several important target proteins involved in cell cycle, apoptosis, senescence and differentiation (13). Additionally, the expression of miR-34a increases with age and is involved in the regulation of telomere maintenance and DNA damage responses, providing a potential mechanism through which telomeres erode during ageing (12). Furthermore, pharmacological inhibition of miR-34a improves cardiac regeneration and function in experimental models of myocardial infarction and in aged mice with heart dysfunction $(11,12)$. Additionally, previous studies have suggested that miR-34a expression was increased in DOXO-treated rat cardiac cells culture medium compared with untreated rat cardiac cells (14). Therefore, modulation of this microRNA may present a potential therapeutic option for cardiac protection during DOXO treatment.

SIRT1 is one of the targets of miR-34a and is a member of the sirtuin family of class III histone deacetylases, which is known to be widely involved in the regulation of cellular gene expression, cell senescence, differentiation and survival $(15,16)$. Previous reports indicate that SIRT1 serves a role in the prevention of various age-related diseases by deacetylating diverse targets, including histones or non-histone substrates (e.g., p53 and fork head proteins) (17,18). Additionally, SIRT1has been reported, in several models of cardiovascular disease, to be involved in the development and progression of heart failure through the regulation of cell senescence-related signaling, with compelling evidence suggesting that molecules that activate SIRT1 cause an anti-senescence effect, leading to cardioprotection $(11,19)$. Additionally, SIRT1 may have the potential to prevent DOXO-induced cardiotoxicity as SIRT1 activation was demonstrated to protect DOXO-exposed cardiac progenitor cells and to re-establish their normal function (20). Consequently, activation of SIRT1 may have great potential in the treatment of DOXO-induced cardiotoxicity. The present study investigated the interaction between MSCs and miR-34a expression, and the involvement of SIRT1 in DOXO-induced cardiotoxicity.

\section{Materials and methods}

Animals. Male Sprague Dawley (SD) rats weighing 60-80 g were cared for in accordance with the published guidelines from the United States National Institutes of Health (21). All animal procedures were approved by the Wenzhou Medical University Institutional Animal Care and Use Committee (Wenzhou, China).

Reagents. The miR-34a mimic andmiR-34a inhibitor were obtained from Invitrogen; Thermo Fisher Scientific, Inc. (Waltham, MA, USA). The Transcriptor First Strand cDNA Synthesis kit, X-treme GENE HP DNA transfection reagent, Telo TAGGG Telomerase PCR ELISA PLUS kit and Fast Start Universal SYBR Master (ROX) were obtained from Roche Diagnostics GmbH (Mannheim, Germany). Rabbit monoclonal antibody against SIRT1 (1:1,000; cat. no. 9475) was purchased from Cell Signaling Technology, Inc. (Danvers, MA, USA). Mouse polyclonal $\beta$-actin antibody was purchased from OriGene Technologies, Inc. (cat. no. TA-09; Rockville, MD, USA). SIRT1 small interfering (si)RNA was obtained from Thermo Fisher Scientific, Inc). MTT and dimethyl sulfoxide (DMSO) were purchased from Sigma-Aldrich; Merck KGaA (Darmstadt, Germany).

Cell culture. Rat embryonic H9c2 myoblasts were obtained from American Type Culture Collection (Manassas, VA, USA). The cells were cultured in Dulbecco's modified Eagle's medium (DMEM), supplemented with $10 \%$ fetal bovine serum (FBS). Both were purchased from Hyclone (GE Healthcare, Chicago, IL, USA).

BM-MSCs were isolated from the femur and tibia of SD rats, as described previously (22). Briefly, bone marrow cells were flushed out from the femur and tibia using $5 \mathrm{ml}$ DMEM/F-12 (Hyclone; GE Healthcare). Next, the red blood cells were lysed in blood cell lysis buffer (Beyotime Institute of Biotechnology, Haimen, China) and discarded, and the remaining cells $\left(5 \times 10^{5}\right)$ were plated into a $25 \mathrm{~cm}^{2}$ flask in $6 \mathrm{ml} \mathrm{DMEM} / \mathrm{F}-12$ supplemented with $10 \% \mathrm{FBS}$ and $1 \%$ penicillin/streptomycin. The cells were cultured at $37^{\circ} \mathrm{C}$ and $5 \% \mathrm{CO}_{2}$. After 3 days in culture, the non-adherent cells were washed away, while the adherent MSCs were maintained in culture. The culture medium was replaced every 3 days. Once the culture reached $80-90 \%$ confluence, the cells were trypsinized and passaged at $2: 3$ or at a dilution of 1:2. All cells used in subsequent assays were passaged 3 to 5 times.

Transwell co-cultures of MSCs-H9c2 and cell treatment. A Transwell system was used to prevent the MSCs from directly contacting the H9c2 cells. MSCs and H9c2 cells were placed into the upper and lower chambers of the Transwell system (density, $1 \times 10^{6}$ cells/well), respectively. The H9c2 cells were pre-treated with DOXO $(0.5 \mu \mathrm{M}$; cat. no. D1515; Sigma-Aldrich; Merck KGaA, Darmstadt, Germany) at $37^{\circ} \mathrm{C}$ for $1 \mathrm{~h}$, as previously described (14). Prior to co-culture, the $\mathrm{H} 9 \mathrm{c} 2$ cells were washed with phosphate-buffered saline. The untreated $\mathrm{H} 9 \mathrm{c} 2$ cells were used as a control.

Cell transfection. Prior to transfection with Lipofectamine ${ }^{\circledR}$ (Invitrogen; Thermo Fisher Scientific, Inc.), MSCs were seeded onto 6-well plates at a density of $1 \times 10^{6}$ cells per well and were incubated at $37^{\circ} \mathrm{C}$ overnight. For overexpression or inhibition of miR-34a, the cells were transfected with amiR-34a mimic or a miR-34a inhibitor (100 $\mathrm{nM})$, and transfection efficiency was analyzed using reverse transcription-quantitative polymerase chain reaction (RT-qPCR) analysis. The interval between transfection and subsequent experimentation was $6 \mathrm{~h}$.

Cell proliferation assay. The rate of cell proliferation was estimated using the Cell Counting Kit-8 (CCK-8) assay, according to the manufacturer's protocol (22). Briefly, $1 \times 10^{5}$ cells were grown on a 96-well plate and were incubated with the CCK-8 solution for $1 \mathrm{~h}$ at $37^{\circ} \mathrm{C}$. Subsequently, the absorbance at $450 \mathrm{~nm}$ was recorded, and a total of three repeats were performed.

MTT assay. The MTT assay was used to determine cell viability as previously described (23). Briefly, $300 \mu \mathrm{l} \mathrm{MTT}$ 
reagent was added to each well $2 \mathrm{~h}$ prior to harvesting. The supernatant was then removed and incubated with $400 \mu 1$ DMSO for $10 \mathrm{~min}$. The absorbance at $540 \mathrm{~nm}$ was recorded using an ELISA plate reader. A total of three repeats were performed.

$R T-q P C R$. The expression levels of several genes were analyzed using RT-qPCR. Briefly, total RNA was extracted from MSCs using TRIzol ${ }^{\circledR}$ reagent (Thermo Fisher Scientific, Inc.) and reverse transcribed using the Transcriptor First Stand cDNA Synthesis kit, according to the manufacturer's protocol. The qPCR was performed using the Fast Start Universal SYBR Master (Roche Diagnostics $\mathrm{GmbH}$ ), and fluorescence qPCR system (Bio-Rad Laboratories, Inc., Hercules, CA, USA). The samples were subjected to 40 cycles of amplification at $95^{\circ} \mathrm{C}$ for $15 \mathrm{sec}$ followed by $64^{\circ} \mathrm{C}$ for $20 \mathrm{sec}$ and $72^{\circ} \mathrm{C}$ for $25 \mathrm{sec}$ using specific primers. The threshold number of cycles $(\mathrm{Cq})$ was set within the exponential phase of the PCR reaction. The $\Delta \mathrm{Cq}$ value for each target gene was calculated by subtracting the $\mathrm{Cq}$ value of GAPDH (internal control) from the target gene, while relative gene expression levels were calculated by comparing the $2^{-\Delta \Delta \mathrm{Cq}}$ values between the control and experimental conditions for each target PCR using the following equation: Relative gene expression $=2^{-(\Delta \mathrm{Cq} \text { sample- } \Delta \mathrm{Cq} \text { control })}(24)$. The primer sequences used to detect the mRNA levels of target genes are listed in Table I.

Western blot analysis. Western blot analysis was performed as previously described (23). Briefly, the cells were ruptured using cell lysis buffer (Beyotime Institute of Biotechnology). The lysates were centrifuged $(12,000 \mathrm{x} \mathrm{g})$ for $5 \mathrm{~min}$ at $4^{\circ} \mathrm{C}$, and the supernatant containing cellular protein was used. For each sample, $20 \mu \mathrm{g}$ total protein was resolved by SDS-PAGE (10\% gel) and transferred onto polyvinylidene difluoride membranes. The membranes were incubated overnight at $4^{\circ} \mathrm{C}$ with primary antibodies, SIRT1 and $\beta$-actin (cat no. 4970; Cell Signaling Technology, Inc.) at a dilution of 1:1,000. The following day, membranes were incubated for $1 \mathrm{~h}$ at $37^{\circ} \mathrm{C}$ with the horseradish peroxidase-conjugated goat anti-rabbit secondary antibody (1:2,000; cat no. 7074; Cell Signaling Technology, Inc.) and were developed using chemiluminescent substrates (BeyoECL Plus; Beyotime Institute of Biotechnology). The stained protein bands were visualizedusing Bio-Rad ChemiDoc XRS equipment and were analyzed using the Quantity One software (both Bio-Rad Laboratories, Inc.).

ELISA. The concentration of secreted vascular endothelial growth factor (VEGF) in the cell culture medium was measured using an ELISA kit (cat. no. RAB0512; Sigma-Aldrich; Merck KGaA). The assays were performed in 96-well microplates according to the manufacturer's protocol. A total of three repeats were performed.

siRNA knockdown. The cells were transfected using X-tremeGENE HP DNA transfection reagent (Roche Diagnostics $\mathrm{GmbH}$ ), according to the manufacturer's protocol. $\mathrm{H} 9 \mathrm{c} 2$ cells were plated onto a 6 -well plate at a density of $1 \times 10^{6}$ cells/well and were treated with X-tremeGENE HP DNA Transfection reagent in a 3:1 ratio of reagent volume to siRNA mass for $20 \mathrm{~min}$. The cells were then transfected
Table I. Primer sequences.

Primer Sequence (5'-3')

p16
Forward

Reverse

p53

Forward

Reverse

miR-34a

Forward

Reverse

Forward

Reverse

Telomere length

Forward

Reverse

\section{GAPDH}

Forward

Reverse

miR-34a mimic

miR-34a inhibitor siRNA-SIRT1

Forward

Reverse

siRNA-NT

Forward

Reverse
SIRT1
GGTCACCGACAGGCATAACTTC'

AAAGGAGGGCTGAGGCCTAA

TCTGTCATCTTCCGTCCCTTCTC

CCGTGCACATAACAGACTTGGCT

AAGGCCACGGATAGGTCCATA CGCTTTGGTGGTTCTGAAAGG

AAGGCCACGGATAGGTCCATA CGCTTTGGTGGTTCTGAAAGG

GGTTTTTGAGGGTGAGGGTGAGG GTGAGGGTGA

TCCCGACTATCCCTATCCCTATCCC TATCCCTATCC

GGCTCTCTGCTCCTCCCTGTT GGCTCTCTGCTCCTCCCTGTT

UGGCAGUGUCUUAGCUGGUUGUU CAACCAGCUAAGACACUGCCAUU UGGCAGUGUCUUAGCUGGUUGUU

GCACCGAUCCUCGAACAAUTT

AUUGUUCGAGGAUCGGUGCTT

UUCUCCGAACGUGUCACGUTT ACGUGACACGUUCGGAGAATT
miR, microRNA; siRNA, small interfering RNA; siRNA-NT, scrambled siRNA; SIRT1, sirtuin 1.

and mixed with $100 \mathrm{nM}$ SIRT1 siRNA, the primer seque nces were as follows: siRNA-SIRT1 GCACCGAUCCUC GAACAAUTTAUUGUUCGAGGAUCGGUGCTT; siRNAnon-targeting (NT) UUCUCCGAACGUGUCACGUTTACG UGACACGUUCGGAGAATT (Thermo Fisher Scientific, Inc.). This mixture was incubated in $2 \mathrm{ml}$ DMEM (aforementioned) medium for $48 \mathrm{~h}$ at $37^{\circ} \mathrm{C}$. Scrambled siRNA (siRNA-NT) was used as the control. The transfection efficiency of siRNA-SIRT1 was assessed by RT-qPCR compared with siRNA-NT. The time period between transfection and subsequent experimentation was $6 \mathrm{~h}$.

Relative telomere length measurement. The quantification of the relative telomere length in $\mathrm{H} 9 \mathrm{c} 2$ cells was performed using qPCR based on a previously established method (25), and GAPDH was used to normalize the expression of target genes. The sequences of the primers used to detect the length of telomeres are listed in Table I. 

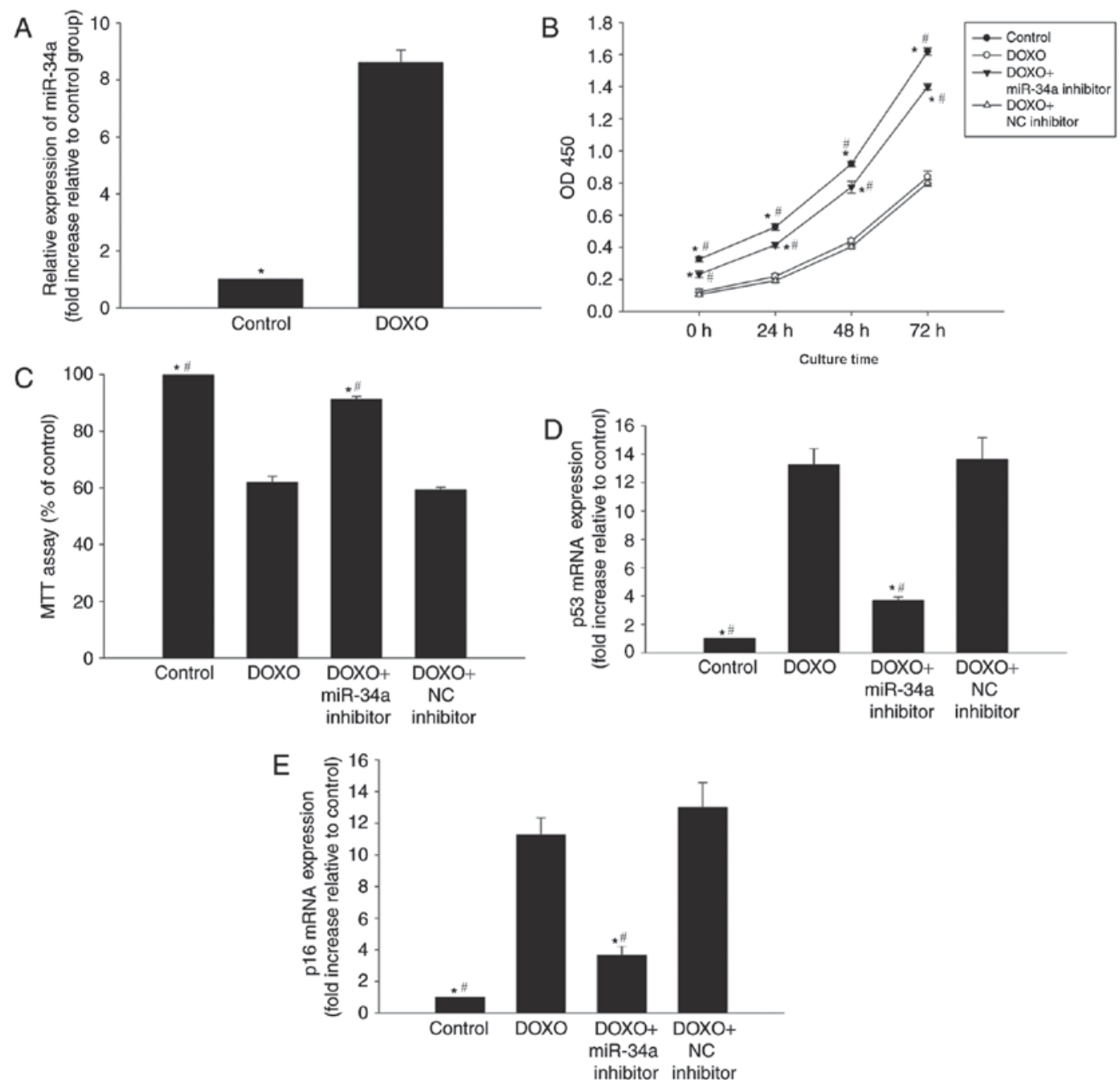

Figure 1. miR-34a is involved in DOXO-induced changes in senescence in H9c2 cells. (A) RT-qPCR analysis of miR-34a mRNA levels in control H9c2 cells and $\mathrm{H} 9 \mathrm{c} 2$ cells treated with DOXO. (B) Proliferation growth curves of H9c2 cells incubated with DOXO, miR-34a inhibitor or NC inhibitor in the presence of $0.5 \mu \mathrm{M}$ DOXO, determined using a Cell Counting Kit-8 assay. (C) DOXO was added to culture medium alone or in combination with the miR-34a inhibitor or NC inhibitor. Cell viability was analyzed using MTT assay. RT-qPCR analysis of (D) p53 and (E) p16 mRNA levels in untreated H9c2 cells and H9c2 cells treated with DOXO or a combination of DOXO and miR-34a inhibitor or NC inhibitor. Data are presented as the mean \pm standard deviation of three independent experiments. "P<0.05 vs. DOXO; " $\mathrm{P}<0.05$ vs. DOXO and NC inhibitor. miR, microRNA; DOXO, doxorubicin; OD, optical density; RT-qPCR, reverse transcription-quantitative polymerase chain reaction; $\mathrm{NC}$, negative control.

Relative telomerase activity measurement (RTA). The telomerase activity of $\mathrm{H} 9 \mathrm{c} 2$ cells was examined using the Telo TAGGG Telomerase PCR ELISA PLUS kit (Sigma-Aldrich; Merck KGaA) according to the manufacturer's protocol. Cell lysates were centrifuged $(12,000 \mathrm{x} \mathrm{g})$ for $20 \mathrm{~min}$ at $4^{\circ} \mathrm{C}$, and $3 \mu \mathrm{l}$ cell extract was used for each telomeric repeat PCR amplification reaction and $3 \mu 1$ inactivated cell lysate was used for Telomeric Repeat Amplification Protocol (TRAP) reactions according to the manufacturer's protocol. Each TRAP reaction was performed with amplification of an internal control from the kit to validate the absence of a PCR inhibitor. Using the ELISA method, the amplified products were immobilized on streptavidin-coated microtiter plates via a biotin-streptavidin interaction. Next, the amplifications were detected using peroxidase-conjugated anti-digoxigenin antibodies provided by the kit. Following the addition of the peroxidase substrate (3,3',5,5'-tetramethylbenzidine), the quantity of TRAP products was determined by measuring absorbance at $450 \mathrm{~nm}$ using a microplate reader.
Statistical analysis. The data are expressed as the mean \pm standard deviation. The differences among multiple groups were determined using one-way analysis of variance followed by Tukeys $s-b(K)$ test, and comparisons between two groups were evaluated by Student's t-test using SPSS software (version 19.0; IBM Corp., Armonk, NY, USA). Cell proliferation data are presented as the mean \pm standard error of the mean. $\mathrm{P}<0.05$ was considered to indicate a statistically significant difference.

\section{Results}

miR-34a is involved in DOXO-induced changes in senescence in $\mathrm{H} 9 \mathrm{c} 2$ cells. To determine whether miR-34a is involved in DOXO-induced cardiac senescence, the expression of miR-34a was evaluated in $\mathrm{H} 9 \mathrm{c} 2$ cells that were exposed to $0.5 \mu \mathrm{M}$ DOXO for $24 \mathrm{~h}$. qPCR analysis revealed a significant upregulation of miR-34a expression in $\mathrm{H} 9 \mathrm{c} 2$ cells following DOXO treatment compared with the control (Fig. 1A). 

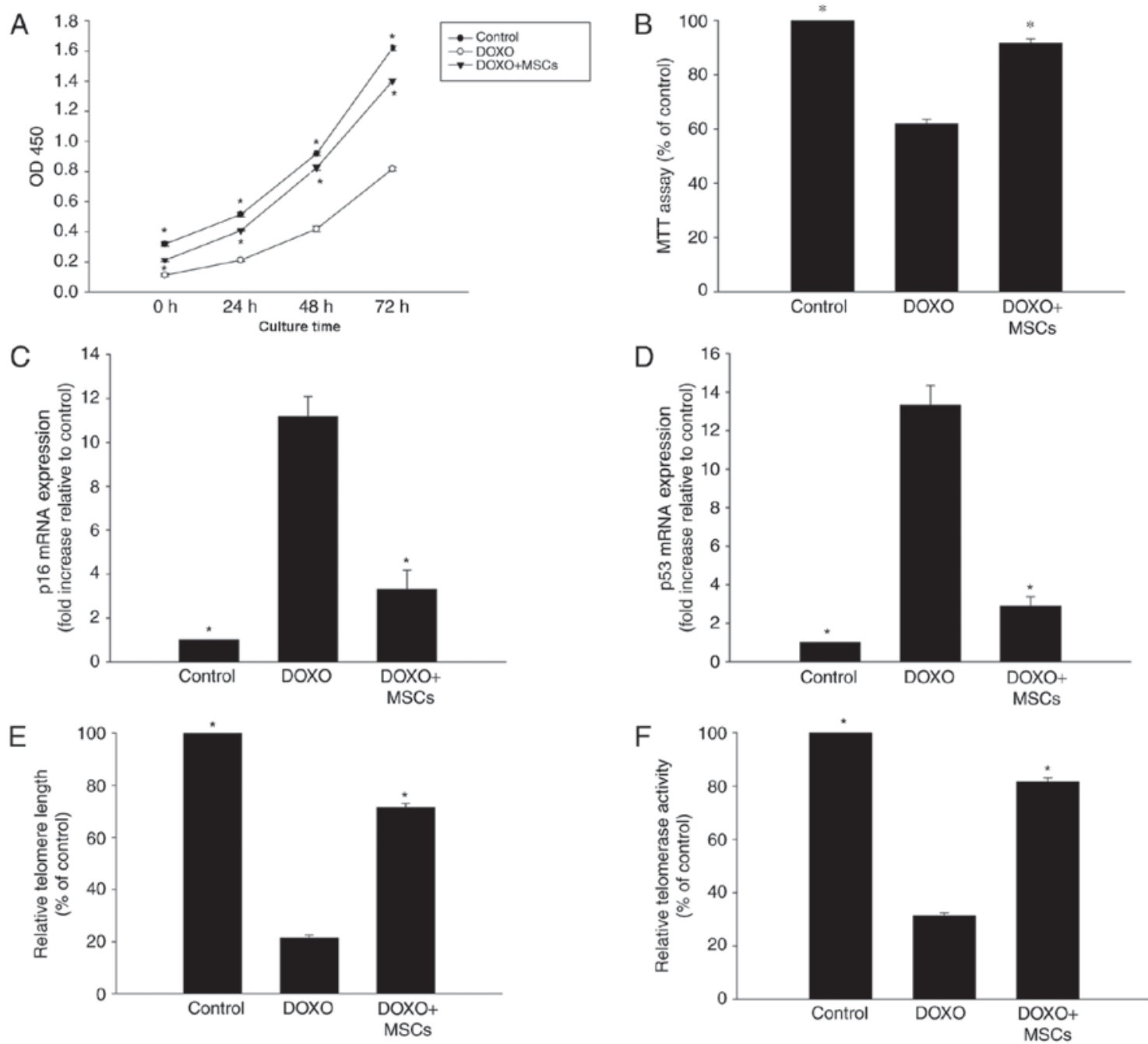

Figure 2. MSCs inhibit DOXO-induced senescence changes in H9c2 cells. In order to investigate the role of the anti-senescence effect of MSCs on DOXO-treated H9c2 cells, a Transwell co-culture system was used. H9c2 cells were incubated with DOXO or co-cultured with MSCs in the presence of DOXO. (A) Cell proliferation was determined by Cell Counting Kit-8 assay, and (B) cell viability was analyzed using MTT assay. The levels of (C) p16 and (D) p53 mRNA were analyzed using RT-qPCR. (E) The length of telomeres in cells was analyzed using RT-qPCR. (F) Relative telomerase activity was measured. Data represent the mean \pm standard deviation of three independent experiments. " $\mathrm{P}<0.05$ vs. DOXO treatment group. MSCs, mesenchymal stem cells; DOXO, doxorubicin; RT-qPCR, reverse transcription-quantitative polymerase chain reaction.

In order to further investigate the role of miR-34a in DOXO-induced senescence in $\mathrm{H} 9 \mathrm{c} 2$ cells, the viability and proliferation of $\mathrm{H} 9 \mathrm{c} 2$ cells were analyzed. The proliferation and percentage of viable cells were decreased following DOXO treatment compared with the control (Fig. 1B and C). Furthermore, the expression of senescence-associated genes, p53 and p16, was markedly increased in the DOXO treatment group compared with the control (Fig. 1D and E). To further investigate the role of miR-34a in this process, it was indicated that the treatment of cells with a miR-34a inhibitor in the presence of DOXO was able to reverse the senescence signature induced by DOXO, while treatment with the $\mathrm{NC}$ inhibitor was not able to reverse the senescence signature induced by DOXO. Additionally, the inhibition of miR-34a resulted in increased proliferation and viability, and was able to reverse the DOXO-induced alterations to senescence-associated genes p53 and p16 (Fig. 1B-E).

MSCs inhibit DOXO-induced senescence changes in H9c2 cells. To investigate the anti-senescence effect of MSCs on
DOXO-treated H9c2 cells, a Transwell co-culture system was employed. As expected, the viability and proliferation of the cells that were treated with DOXO and co-cultured with MSCs were significantly higher compared with cells only treated with DOXO (Fig. 2A and B). Additionally, the expression of p53 and p16 was significantly decreased in cells that were exposed to DOXO and co-cultured with MSCs compared with cells that were only treated with DOXO (Fig. 2C and D). Furthermore, it was observed that DOXO treatment induced shortening of telomeres, which was also associated with a decrease in telomerase activity. However, the addition of MSCs to the co-culture system was able to partly reverse these DOXO-induced alterations (Fig. 2E and F).

MSCs induce anti-senescence by inhibiting miR-34a expression. In order to determine whether or not MSCs induce an anti-senescence effect by inhibiting the expression of miR-34a, the expression of miR-34a in cells that were co-cultured with MSCs was assessed. As indicated in Fig. 3A, in the presence of DOXO, co-culture with MSCs 

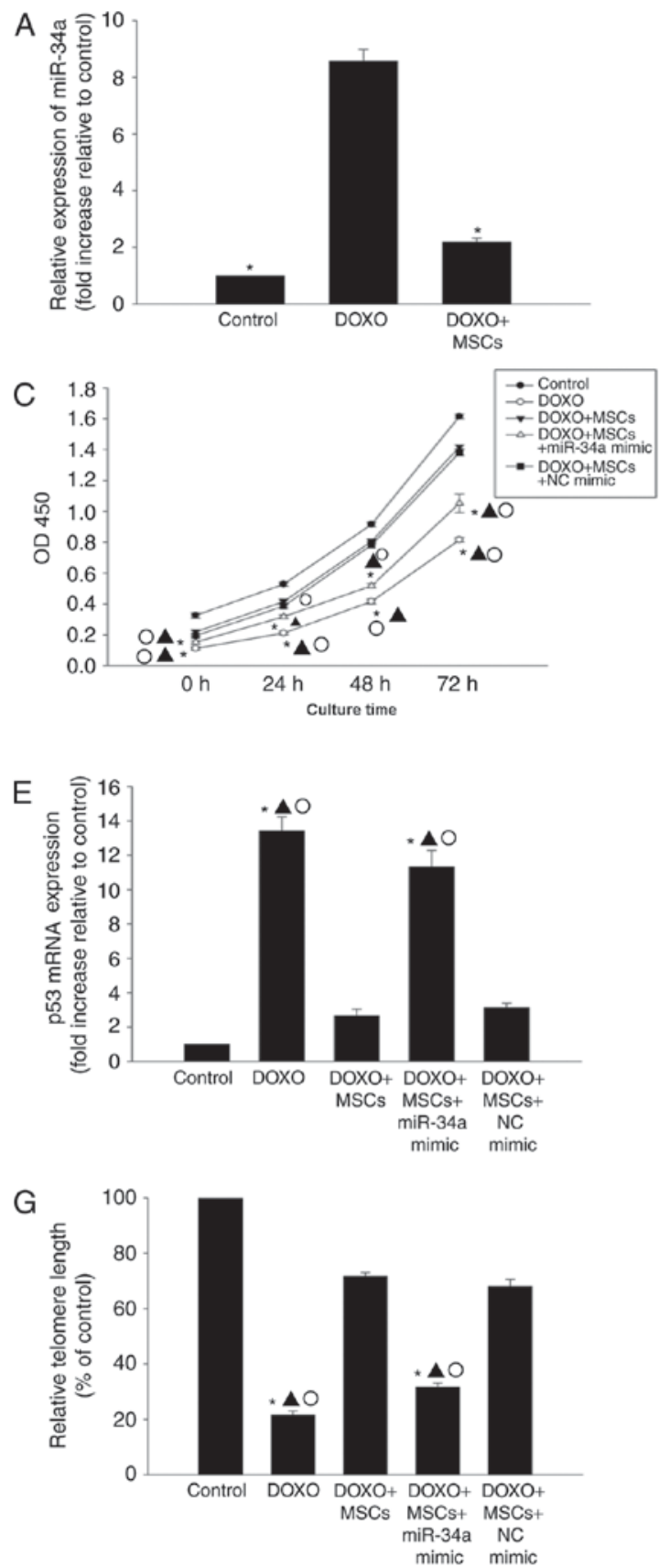
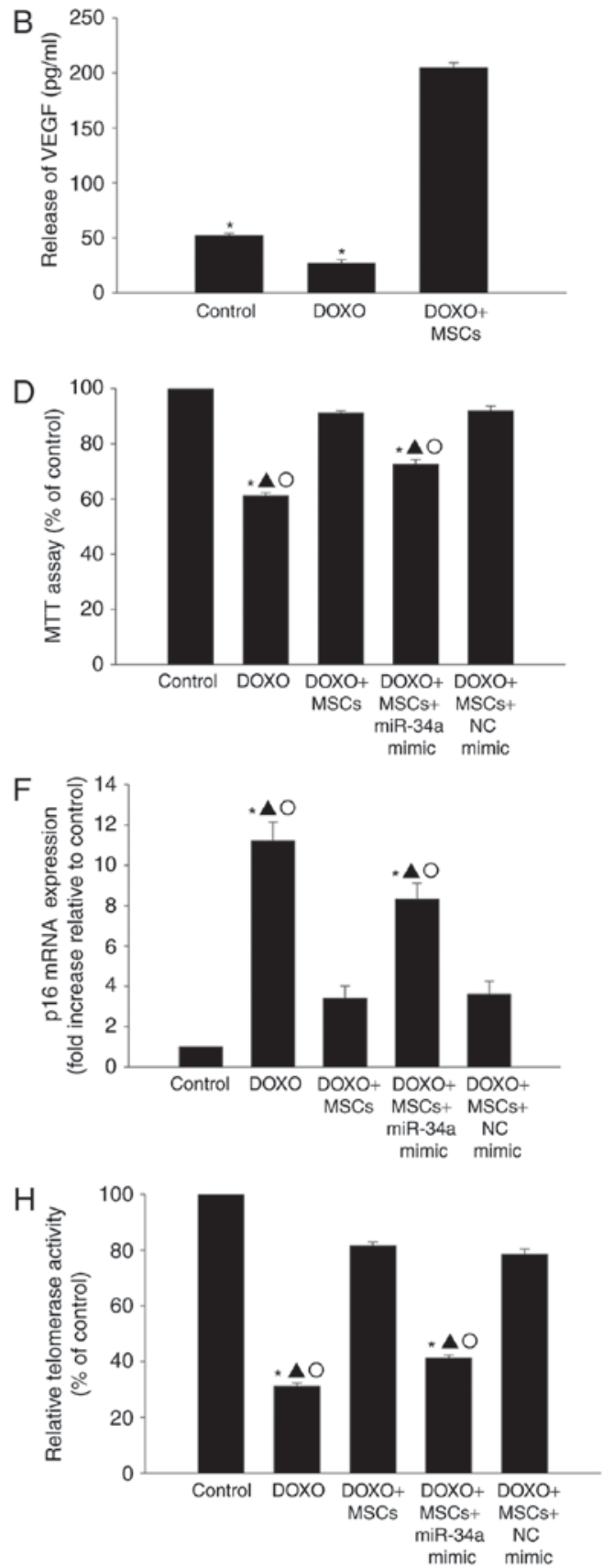

Figure 3. MSCs induce anti-senescence by inhibiting miR-34a expression. (A) RT-qPCR analysis of the levels of miR-34a mRNA in H9c2 cells incubated with DOXO or co-cultured with MSCs in the presence of DOXO. Each data point represents the mean \pm standard deviation of three independent experiments. ${ }^{*} \mathrm{P}<0.05$ vs. DOXO. (B) ELISA analysis of the levels of VEGF released from H9c2 cells that were incubated with DOXO or co-cultured with MSCs. Each data point represents the mean \pm standard deviation of three independent experiments. $\mathrm{P}<0.05$ vs. DOXO+MSCs. (C) Proliferation was determined using Cell Counting Kit-8 assay in several treatment groups: i) DOXO-treated H9c2 cells; ii) co-culture with MSCs and treatment with DOXO; iii) co-culture with MSCs and transfection with a miR-34a mimic in the presence of DOXO and iv) co-culture with MSCs and transfection with NC mimic in the presence of DOXO. (D) The viability of H9c2 cells was analyzed using MTT assay. The analysis of (E) p53, (F) p16 mRNA levels and (G) telomere length was performed using RT-qPCR. (H) The relative telomerase activity was measured. Each data point represents the mean \pm standard deviationof three independent experiments. ${ }^{*} \mathrm{P}<0.05$ vs. control; ${ }^{\wedge} \mathrm{P}<0.05$ vs. DOXO+MSCs; ${ }^{\circ} \mathrm{P}<0.05$ vs. DOXO+MSCs+NC mimic. MSCs, mesenchymal stem cells; miR, microRNA; RT-qPCR, reverse transcription-quantitative polymerase chain reaction; DOXO, doxorubicin; VEGF, vascular endothelial growth factor.

was able to significantly decrease the expression of miR-34a. Additionally, co-culture with MSCs was able to markedly increased the release of VEGF when compared with DOXO treatment only (Fig. 3B).

In the presence of DOXO, co-culture with MSCs was able to improve the proliferation and viability of $\mathrm{H} 9 \mathrm{c} 2$ cells compared with DOXO treatment only (Fig. 3C and D), and reduce the expression of p53 and p16 (Fig. 3E and F).
Additionally, DOXO-induced alterations in telomere length and activity were ameliorated when co-cultured with MSCs (Fig. 3G and H). To assess if the anti-senescence effect was mediated by the inhibition of miR-34a, transfection of a miR-34a mimic was performed to induce the overexpression of miR-34a (data not shown). The overexpression of miR-34a was able to diminish the anti-senescence efficiency of MSCs, resulting in decreased cell viability and proliferation, an 
A
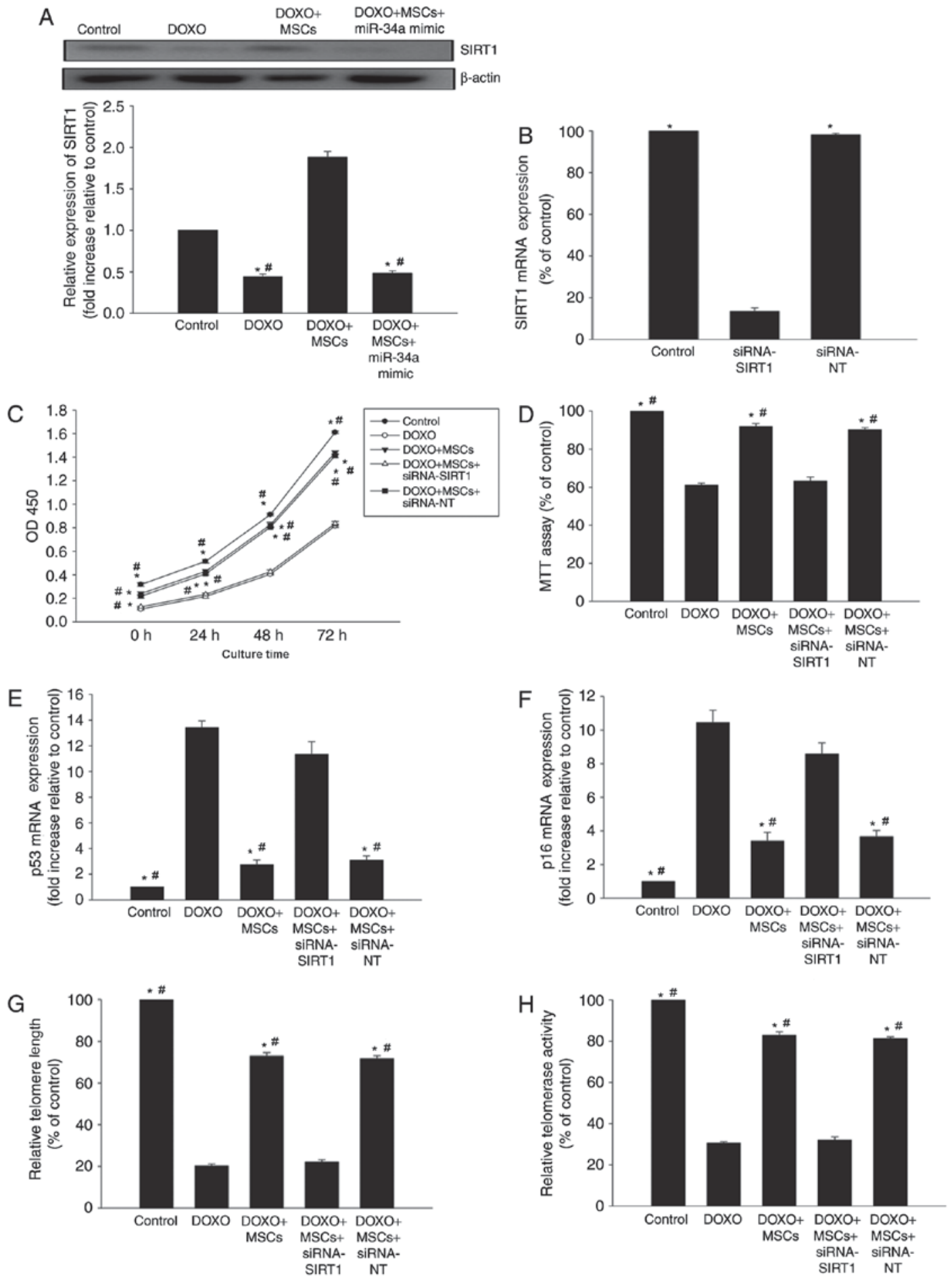

Figure 4. miR-34a-SIRT1 axis serves a role in MSC-mediated anti-senescence effect on DOXO-treated H9c2 cells. (A) The expression of SIRT1 was analyzed by western blot analysis in $\mathrm{H} 9 \mathrm{c} 2$ cells, in the presence of DOXO, MSCs or a miR-34a mimic. Each column represents the mean \pm standard deviation of three independent experiments. ${ }^{*} \mathrm{P}<0.05$ vs. control; ${ }^{\#} \mathrm{P}<0.05$ vs. DOXO+MSCs. (B) RT-qPCR analysis of SIRT1 expression in non-transfected H9c2 cells and in $\mathrm{H} 9 \mathrm{c} 2$ cells transfected with siRNA-SIRT1 or siRNA-NT. Each column represents the mean \pm standard deviation of three independent experiments. $\mathrm{P}<0.05$ vs. siRNA-SIRT1; (C) Proliferation growth curves were determined using Cell Counting Kit-8 assay in DOXO-treated H9c2 cells, H9c2 cells co-cultured with MSCs in the presence of DOXO, and H9c2 cells co-cultured with MSCs transfected with siRNA-SIRT1 or siRNA-NT in the presence of DOXO. (D) Cell viability was analyzed using MTT assay. Each column represents the mean \pm standard deviation of three independent experiments. RT-qPCR analysis of (E) p53, (F) p16 mRNA levels and (G) telomere length. (H) Relative telomerase activity was analyzed. Each column represents the mean \pm standard deviation from three independent experiments. "P<0.05 vs. DOXO; ${ }^{\mathrm{P}}<0.05$ vs. DOXO+MSCs+siRNA-SIRT1. miR, microRNA; MSCs, mesenchymal stem cells; DOXO, doxorubicin; RT-qPCR, reverse transcription-quantitative polymerase chain reaction; siRNA, small interfering RNA; NT, non-transfected; siRNA, small interfering RNA.

increase in expression of $\mathrm{p} 53$ and $\mathrm{p} 16$, and shortened telomere length, which corresponded with a decrease in telomerase activity (Fig. 3C-H).
miR-34a-SIRT1 axis serves a role in the anti-senescence effect of MSCs on DOXO-treated H9c2 cells. SIRT1, one of several miR-34a targets, is a member of the sirtuin family of class 
III histone deacetylases (26). In the presence of DOXO, the expression of SIRT1 decreased in H9c2 cells, whilst co-culture with MSCs increased the expression of SIRT1. However, the overexpression of miR-34a diminished the effect of MSCs on SIRT1 expression (Fig. 4A).

Next, siRNA-SIRT1 was used to silence the expression of SIRT1 (Fig. 4B). The knockdown of SIRT1 was able to decrease the proliferation of $\mathrm{H} 9 \mathrm{c} 2$ cells starting from $24 \mathrm{~h}$ of treatment, and this effect continued for $\geq 72 \mathrm{~h}$. The knockdown of SIRT1 was able to decrease cell viability compared with siRNA-NT controls (Fig. 4C and D). Additionally, abolishing SIRT1 rescued the previously observed decrease in p53 and p16 caused by co-culturing with MSCs (Fig. 4E and F), and abolished the anti-senescence effects of MSCs on telomere length and activity (Fig. 4G and H).

\section{Discussion}

The survival rate of patients with cancer continues to increase. However, there are new concerns over the long-term effects of anticancer treatments and measures to reduce the negative effects of such treatments are an emerging area of investigation (6).

DOXO, which belongs to the anthracycline family, has been demonstrated to be effective in numerous types of tissue-derived cancer, including tumors of the breast, lung, stomach, bladder and skin $(27,28)$. Despite the antitumor properties of DOXO, its cardiotoxic effects have severely restricted its clinical use (5). Pharmacokinetic studies have demonstrated that DOXO induces cardiac toxicity though multiple pathways $(1,14)$. Inhibition of the DNA replication process, leading to the generation of oxidative stress, which progressively causes cardiac cell senescence, is one potential mechanism for DOXO-induced cardiac toxicity $(4,29)$. Current efforts to prevent cardiac toxicity in patients with cancer cannot guarantee permanent cardiac protection.

One of the main limitations in the prevention of cancer therapy-induced cardiac stress is the inability to ameliorate endogenous cardiac cell senescence $(6,30)$. The results of the present study suggest that co-culturing with MSCs may effectively rescue $\mathrm{H} 9 \mathrm{c} 2$ cells treated with DOXO. Furthermore, it was demonstrated that this function is mediated through inhibition of miR-34a and the subsequent activation of SIRT1, leading to increased cell proliferation and viability, decreased expression of senescence-associated genes p53 and p16, and an increase in telomere length and telomerase activity. Taken together, the results of the present study suggest that MSCs may be a promising candidate for the treatment of DOXO-induced cardiomyopathy.

MSC-based therapies have huge potential in the treatment of cardiovascular diseases (31), and MSCs possess a number of characteristics that make them a suitable tool for the treatment of DOXO-induced cardiomyopathy (6). MSCs secrete paracrine factors, including VEGF, insulin-like growth factor and basic fibroblast growth factor, with proliferative and anti-apoptotic properties, and are able to modulate various senescenceassociated signaling pathways involved in the cardiac regenerative process (32). A previous study demonstrated that MSCs have a cytoprotective effect on the anoxia-induced apoptosis of $\mathrm{H} 9 \mathrm{C} 2$ cells. The underlying mechanisms may be associated with increased levels of VEGF mRNA and protein (33).
The same results were observed in the present study, with an increase in VEGF expression in H9c2 cells following co-culture with MSCs and DOXO when compared with control and H9c2 cells treated with only DOXO. Furthermore, MSCs are known to have anti-inflammatory properties, with a previous study confirming that, through modulation of the inflammatory microenvironment, MSCs manage elevated tissue inflammation stress by suppressing the immune response and modifying the inflammatory micro environment (34). In line with this, by co-culturing $\mathrm{H} 9 \mathrm{c} 2$ cells with MSCs, the present study confirmed that inhibiting cellular senescence may be an important target in DOXO-induced cardiomyopathy.

The therapeutic effects of miR-34a in the heart have been previously examined in various models of cardiac disease, including myocardial infarction (11). In keeping with the findings of the present study, miR-34a was reported to be highly expressed in damaged heart tissue, and the loss of miR-34a was revealed to improve cardiac function and reduce cell death in ageing hearts (12). The results of the present study suggest that DOXO-induced elevated expression of miR-34a is associated with the senescent state of $\mathrm{H} 9 \mathrm{c} 2$ cells. Previous studies have reported that miR-34a may also confer part of its biological activity through conditioned medium from cell culture, as well as the presence in body fluids (35). The present study used a Transwell co-culture system. A decrease in miR-34a expression in $\mathrm{H} 9 \mathrm{c} 2$ cells and ameliorated cellular senescence induced by treatment with DOXO was observed. However, these effects were diminished when a miR-34a mimic was used to overexpress miR-34a, confirming that MSCs modulate the anti-senescence effect through inhibition of miR-34a in DOXO-induced cardiac toxicity.

SIRT1, an NAD-dependent deacetylase, has been implicated in improving metabolism and the prevention of various age-related diseases (36). As an important direct target of miR-34a, SIRT1 has previously been associated with cellular survival, apoptosis and senescence (37). In line with this, the miR-34a-SIRT axis has been implicated in age-related heart diseases. Previous studies have suggested that activation of the miR-34a-SIRT axis may be a critical regulator of cardiac repair and regeneration post myocardial infarction in the aged heart and thus, modulation of SIRT1 may be harnessed for cardiac repair in the aged myocardium $(11,26)$. The present study demonstrated that, in the presence of DOXO, expression of SIRT1 decreases, while co-culturing with MSCs rescues this effect. To further confirm this finding, siRNA-SIRT1 was used to silence SIRT1, which was revealed to abolish the anti-senescence effect of MSCs.

As an important protein associated with age, SIRT1 functions by deacetylating diverse target histones, $\mathrm{H} 1, \mathrm{H} 3$ and $\mathrm{H} 4$, as well as a large number of non-histone substrates, including p53 and fork head proteins $(18,38,39)$. The most commonly accepted miR34a-SIRT1-p53 axis induced by miR-34a in cell senescence is in a positive feedback loop, where miR-34a inhibits SIRT1, which activates p53, which in turn activates miR-34a (40). Exogenous stress activates miR-34a, which inhibits SIRT1 expression and, in turn, activates p53 (18). It is well established that cellular senescence is the main process that leads to the functional failure of stem cells contributing to the onset and progression of diseases. A recent study suggested that the activation of the miR-34a/SIRT1/p53 signaling pathway 
contributes to age-related hearing loss (41). Furthermore, the results of the present study suggest that MSCs inhibit the increase in expression of p53 induced by DOXO and that the overexpression of miR-34a or silencing of SIRT1 may weaken the effect of MSCs on p53 expression. Future studies by the authors will investigate whether the DOXO-induced reduction in $\mathrm{H} 9 \mathrm{c} 2$ viability was due to an increase in apoptosis or cell cycle arrest, and whether MSC co-culture may modulate these processes.

Telomeres are specialized nucleoprotein structures that protect the ends of eukaryotic chromosomes from unscheduled DNA repair (42). In vertebrates, telomeres are constituted by TTAGGG tandem repeats, which serve a pivotal role in the regulation of telomere length and protection of chromosome ends (43). As an important mark of senescence, telomeres are also closely associated with heart disease. A previous study demonstrated that shortened telomeres are associated with heart failure (44). Additionally, a previous study confirmed that decreased telomere length was associated with vascular aging, leading to hypertension (45). Furthermore, it has been reported that SIRT1 attenuates telomere attrition in vivo and was recruited at telomeres in induced pluripotent stem cells (46). The present study revealed that treatment with DOXO induced telomere shortening and decreased telomerase activity, while MSCs alleviated these alterations and silencing SIRT1 reversed the MSC-induced changes.

The present study demonstrated that MSCs may effectively rejuvenate senescent $\mathrm{H} 9 \mathrm{c} 2$ cells. The results of the present study suggest that MSCs protect $\mathrm{H} 9 \mathrm{c} 2$ cells from DOXO-induced senescence via inhibition of miR-34a, leading to the activation of SIRT1, which elongates telomere length and increases telomerase activity. The findings presented here suggest that exogenous interventions with MSCs leading to activation of associated signaling pathways in the senescent heart may be useful in reducing DOXO-induced cardiac damage in patients receiving chemotherapy.

\section{Acknowledgements}

Not applicable.

\section{Funding}

The present study was supported by the National Natural Science Foundation of China (grant no. 81500261), the Science and Technology Planning Project of Wenzhou (grant no. Y20160125) and the Medical Science and Technology Project of Zhejiang Province (grant no. 2018236627).

\section{Availability of data and materials}

All data generated or analyzed during the present study are included in the published article.

\section{Authors' contributions}

WZX made substantial contributions to the acquisition of data, analysis and interpretation of data; and $\mathrm{MH}$ was involved in conception and design, drafting the manuscript and revising it critically for important intellectual content.

\section{Ethics approval and consent to participate}

All animal procedures were approved by the Wenzhou Medical University Institutional Animal Care and Use Committee (Wenzhou, China).

\section{Consent for publication}

Not applicable.

\section{Competing interests}

The authors declare that there are no competing interests.

\section{References}

1. Rochette L, Guenancia C, Gudjoncik A, Hachet O, Zeller M, Cottin Y and Vergely C: Anthracyclines/trastuzumab: New aspects of cardiotoxicity and molecular mechanisms. Trends Pharmacol Sci 36: 326-348, 2015.

2. Stone JB and DeAngelis LM: Cancer-treatment-induced neurotoxicity-focus on newer treatments. Nat Rev Clin Oncol 13: 92-105, 2016

3. Suter TM and Ewer MS: Cancer drugs and the heart: Importance and management. Eur Heart J 34: 1102-1111, 2013.

4. Franco VI, Henkel JM, Miller TL and Lipshultz SE: Cardiovascular effects in childhood cancer survivors treated with anthracyclines. Cardiol Res Pract 2011: 134679, 2011.

5. Volkova M and Russell R III: Anthracycline cardiotoxicity: Prevalence, pathogenesis and treatment. Curr Cardiol Rev 7: 214-220, 2011.

6. Ezquer F, Gutiérrez J, Ezquer M, Caglevic C, Salgado HC and Calligaris SD: Mesenchymal stem cell therapy for doxorubicin cardiomyopathy: Hopes and fears. Stem Cell Res Ther 6: 116, 2015.

7. Yu Q, Li Q, Na R, Li X, Liu B, Meng L, Liutong H, Fang W, Zhu $\mathrm{N}$ and Zheng $\mathrm{X}$ : Impact of repeated intravenous bone marrow mesenchymal stem cells infusion on myocardial collagen network remodeling in a rat model of doxorubicin-induced dilated cardiomyopathy. Mol Cell Biochem 387: 279-285, 2014.

8. Garbade J, Dhein S, Lipinski C, Aupperle H, Arsalan M, Borger MA, Barten MJ, Lehmann S, Walther T and Mohr FW: Bone marrow-derived stem cells attenuate impaired contractility and enhance capillary density in a rabbit model of Doxorubicin-induced failing hearts. J Card Surg 24: 591-599, 2009.

9. Eulalio A, Mano M,Dal Ferro M,Zentilin L, Sinagra G,Zacchigna S and Giacca M: Functional screening identifies miRNAs inducing cardiac regeneration. Nature 492: 376-381, 2012.

10. Seeger FH, Zeiher AM and Dimmeler S: MicroRNAs in stem cell function and regenerative therapy of the heart. Arterioscler Thromb Vasc Biol 33: 1739-1746, 2013.

11. Yang Y, Cheng HW, Qiu Y, Dupee D, Noonan M, Lin YD, Fisch S, Unno K, Sereti KI and Liao R: MicroRNA-34a plays a key role in cardiac repair and regeneration following myocardial infarction. Circ Res 117: 450-459, 2015.

12. Boon RA, Iekushi K, Lechner S, Seeger T, Fischer A, Heydt S, Kaluza D, Tréguer K, Carmona G, Bonauer A, et al: MicroRNA-34a regulates cardiac ageing and function. Nature 495: 107-110, 2013.

13. Chen F and Hu SJ: Effect of microRNA-34a in cell cycle, differentiation, and apoptosis: A review. J Biochem Mol Toxicol 26: 79-86, 2012.

14. Piegari E, Russo R, Cappetta D, Esposito G, Urbanek K, Dell'Aversana C, Altucci L, Berrino L, Rossi F and De Angelis A: MicroRNA-34a regulates doxorubicin-induced cardiotoxicity in rat. Oncotarget 7: 62312-62326, 2016.

15. Haigis MC and Sinclair DA: Mammalian sirtuins: Biological insights and disease relevance. Annu Rev Pathol 5: 253-295, 2010.

16. Morris BJ: Seven sirtuins for seven deadly diseases of aging. Free Radic Biol Med 56: 133-171, 2013.

17. Deng CX: SIRT1, is it a tumor promoter or tumor suppressor? Int J Biol Sci 5: 147-152, 2009. 
18. Luo J, Nikolaev AY, Imai S, Chen D, Su F, Shiloh A, Guarente L and Gu W: Negative control of p53 by Sir2alpha promotes cell survival under stress. Cell 107: 137-148, 2001.

19. Yang G, Zhang X, Weng X, Liang P, Dai X, Zeng S, Xu H, Huan H, Fang M, Li Y, et al: SUV39H1 mediated SIRT1 trans-repression contributes to cardiac ischemia-reperfusion injury. Basic Res Cardiol 112: 22, 2017.

20. De Angelis A, Piegari E, Cappetta D, Russo R, Esposito G, Ciuffreda LP, Ferraiolo FA, Frati C, Fagnoni F, Berrino L, et al: SIRT1 activation rescues doxorubicin-induced loss of functional competence of human cardiac progenitor cells. Int J Cardiol 189: 30-44, 2015

21. Liu WH, Liu JJ, Wu J, Zhang LL, Liu F, Yin L, Zhang MM and Yu B: Novel mechanism of inhibition of dendritic cells maturation by mesenchymal stem cells via interleukin-10 and the JAK1/STAT3 signaling pathway. PLoS One 8: e55487, 2013.

22. Xia W, Zhang F, Xie C, Jiang M and Hou M: Macrophage migration inhibitory factor confers resistance to senescence through CD74-dependent AMPK-FOXO3a signaling in mesenchymal stem cells. Stem Cell Res Ther 6: 82, 2015.

23. Xia W, Xie C, Jiang M and Hou M: Improved survival of mesenchymal stem cells by macrophage migration inhibitory factor. Mol Cell Biochem 404: 11-24, 2015.

24. Livak KJ and Schmittgen TD: Analysis of relative gene expression data using real-time quantitative PCR and the 2(-Delta Delta C(T)) method. Methods 25: 402-408, 2001.

25. Crepin T, Carron C, Roubiou C, Gaugler B, Gaiffe E, Simula-Faivre D, Ferrand C, Tiberghien P, Chalopin JM, Moulin B, et al: ATG-induced accelerated immune senescence: Clinical implications in renal transplant recipients. Am J Transplant 15: 1028-1038, 2015.

26. Zhang F, Cui J, Liu X, Lv B, Liu X, Xie Z and Yu B: Roles of microRNA-34a targeting SIRT1 in mesenchymal stem cells. Stem Cell Res Ther 6: 195, 2015.

27. Bao Y, Yin M, Hu X, Zhuang X, Sun Y, Guo Y, Tan S and Zhang Z: A safe, simple and efficient doxorubicin prodrug hybrid micelle for overcoming tumor multidrug resistance and targeting delivery. J Control Release 235: 182-194, 2016

28. Tanaka M, Koul D, Davies MA, Liebert M, Steck PA and Grossman HB: MMAC1/PTEN inhibits cell growth and induces chemosensitivity to doxorubicin in human bladder cancer cells. Oncogene 19: 5406-5412, 2000.

29. Liu J, Mao W, Ding B and Liang CS: ERKs/p53 signal transduction pathway is involved in doxorubicin-induced apoptosis in $\mathrm{H} 9 \mathrm{c} 2$ cells and cardiomyocytes. Am J Physiol Heart Circ Physiol 295: H1956-H1965, 2008.

30. Burridge PW, Li YF, Matsa E, Wu H, Ong SG, Sharma A, Holmström A, Chang AC, Coronado MJ, Ebert AD, et al: Human induced pluripotent stem cell-derived cardiomyocytes recapitulate the predilection of breast cancer patients to doxorubicin-induced cardiotoxicity. Nat Med 22: 547-556, 2016.

31. Golpanian S, Wolf A, Hatzistergos KE and Hare JM: Rebuilding the damaged heart: Mesenchymal stem cells, cell-based therapy, and engineered heart tissue. Physiol Rev 96: 1127-1168, 2016

32. Samper E, Diez-Juan A, Montero JA and Sepúlveda P: Cardiac cell therapy: Boosting mesenchymal stem cells effects. Stem Cell Rev 9: 266-280, 2013
33. Zhao SL, Zhang YJ, Li MH, Zhang XL and Chen SL: Mesenchymal stem cells with overexpression of midkine enhance cell survival and attenuate cardiac dysfunction in a rat model of myocardial infarction. Stem Cell Res Ther 5: 37, 2014.

34. Wang Y, Chen X, Cao W and Shi Y: Plasticity of mesenchymal stem cells in immunomodulation: Pathological and therapeutic implications. Nat Immunol 15: 1009-1016, 2014.

35. Creemers EE, Tijsen AJ and Pinto YM: Circulating microRNAs: Novel biomarkers and extracellular communicators in cardiovascular disease? Circ Res 110: 483-495, 2012.

36. Wang H, Han L, Zhao G, Shen H, Wang P, Sun Z, Xu C, Su Y, Li G, Tong T and Chen J: hnRNP A1 antagonizes cellular senescence and senescence-associated secretory phenotype via regulation of SIRT1 mRNA stability. Aging cell: Sep 9, 2016 (Epub ahead of print).

37. McCubbrey AL, Nelson JD, Stolberg VR, Blakely PK, McCloskey L, Janssen WJ, Freeman CM and Curtis JL: MicroRNA-34a negatively regulates efferocytosis by tissue macrophages in part via SIRT1 196: 1366-1375, 2016

38. Brunet A, Sweeney LB, Sturgill JF, Chua KF, Greer PL, Lin Y, Tran H, Ross SE, Mostoslavsky R, Cohen HY, et al: Stress-dependent regulation of FOXO transcription factors by the SIRT1 deacetylase. Science 303: 2011-2015, 2004

39. Motta MC, Divecha N, Lemieux M, Kamel C, Chen D, Gu W, Bultsma Y, McBurney M and Guarente L: Mammalian SIRT1 represses forkhead transcription factors. Cell 116: 551-563, 2004.

40. Zhao T, Li J and Chen AF: MicroRNA-34a induces endothelial progenitor cell senescence and impedes its angiogenesis via suppressing silent information regulator 1. Am J Physiol Endocrinol Metab 299: E110-E116, 2010.

41. Xiong H, Pang J, Yang H, Dai M, Liu Y, Ou Y, Huang Q, Chen S, Zhang Z, Xu Y, et al: Activation of miR-34a/SIRT1/p53 signaling contributes to cochlear hair cell apoptosis: Implications for age-related hearing loss. Neurobiol Aging 36: 1692-1701, 2015.

42. Chan SR and Blackburn EH: Telomeres and telomerase. Philos Trans R Soc Lond B Biol Sci 359: 109-121, 2004.

43. de Lange T: Shelterin: The protein complex that shapes and safeguards human telomeres. Genes Dev 19: 2100-2110, 2005.

44. Mourkioti F, Kustan J, Kraft P, Day JW, Zhao MM, Kost-Alimova M, Protopopov A, DePinho RA, Bernstein D, Meeker AK and Blau HM: Role of telomere dysfunction in cardiac failure in Duchenne muscular dystrophy. Nat Cell Biol 15: 895-904, 2013.

45. Boe AE, Eren M, Murphy SB, Kamide CE, Ichimura A, Terry D, McAnally D, Smith LH, Miyata T and Vaughan DE: Plasminogen activator inhibitor-1 antagonist TM5441 attenuates Nomega-nitro-L-arginine methyl ester-induced hypertension and vascular senescence. Circulation 128: 2318-2324, 2013.

46. De Bonis ML, Ortega S and Blasco MA: SIRT1 is necessary for proficient telomere elongation and genomic stability of induced pluripotent stem cells. Stem Cell Reports 2: 690-706, 2014. 